Research, Society and Development, v. 9, n. 8, e105985363, 2020

(CC BY 4.0) | ISSN 2525-3409 | DOI: http://dx.doi.org/10.33448/rsd-v9i8.5363

\title{
A influência das amizades no comportamento e na saúde dos adolescentes
}

The influence of friendships on adolescent's behavior and health

La influencia de los amigos sobre el comportamiento y la salud de los adolescentes

Recebido: 06/06/2020 | Revisado: 09/06/2020 | Aceito: 12/06/2020 | Publicado: 25/06/2020

\section{Eloisa da Fonseca Rodrigues}

ORCID: https://orcid.org/0000-0002-5052-4086

Universidade Federal do Rio Grande, Brasil

E-mail: eloisadafonsecarodrigues@yahoo.com.br

Giovana Calcagno Gomes

ORCID: https://orcid.org/0000-0002-2464-1537

Universidade Federal do Rio Grande, Brasil

E-mail: giovanacalcagno@ furg.br

Luciano Garcia Lourenção

ORCID: https://orcid.org/0000-0002-1240-4702

Universidade Federal do Rio Grande, Brasil

E-mail: Luciano.famerp@gmail.com

Simone Quadros Alvarez

ORCID: https://orcid.org/0000-0002-0501-7202

Serviço Nacional do Comércio, Brasil

E-mail: simone.alvarez@ibest.com

Aline Campelo Pintanel

ORCID: https://orcid.org/0000-0002-5076-8902

Universidade Federal do Rio Grande, Brasil

E-mail: acpintanel@ hotmail.com

Juliane Portella Ribeiro

ORCID: https://orcid.org/0000-0002-1882-6762

Universidade Federal de Pelotas, Brasil

E-mail: ju_ribeiro1985@hotmail.com

\section{Resumo}

Objetivo: Identificar como os adolescentes interpretam e reagem diante de suas relações de amizade e quais suas repercussões no bem-estar e saúde nesta fase da vida. Método: Trata-se 
de um estudo transversal de abordagem qualitativa, exploratório e descritivo realizado no período de junho a julho de 2019. O cenário da pesquisa foi uma escola localizada em município do extremo sul do estado do Rio Grande do Sul, que se dedica a oferta de cursos pré-profissionalizantes a estudantes adolescentes em situação de vulnerabilidade social, econômica e ambiental. Resultados: Participaram do estudo 30 adolescentes. Após análise dos temas originados nas entrevistas emergiram as seguintes categorias: Relações de amizade saudáveis e promotoras de afeto, empatia e confiança; Confiança: a necessidade de uma base segura nas interações e Amizades disfuncionais: um risco para a saúde do adolescente. Considerações Finais: As relações interpessoais durante a adolescência são permeadas de singularidades e sutilezas e os indivíduos estão mais sensíveis a críticas e desaprovação por seus pares. Atitudes negativas como rejeição e negligência têm caráter de maior exposição a comportamentos que comprometem sobremaneira seu desenvolvimento saudável.

Palavras-chave: Adolescente; Comportamento do adolescente; Saúde; Enfermagem.

\begin{abstract}
Objective: To identify how adolescents interpret and react to their friendly relationships and what are their repercussions on welfare and health at this life stage. Method: This is a crosssectional study with a qualitative, exploratory and descriptive approach conducted from June to July 2019. The research setting was a school located in a town in the extreme south of the Rio Grande do Sul State, which is dedicated to offering pre-professional courses to adolescent students in situations of social, economic and environmental vulnerability. Results: This study was attended by 30 adolescents. After analyzing the topics originated in the interviews, we raised the following categories: Healthy friendship relationships that promote affection and empathy; Trust: the need for a safe basis in interactions and dysfunctional friendships: a risk for adolescent's health. Final Considerations: Interpersonal relationships during adolescence are permeated with singularities and nuances, where individuals are more sensitive to criticism and disapproval by their peers. Negative attitudes of rejection and neglect have the character of greater exposure to behaviors that greatly undermine their healthy development.
\end{abstract}

Keywords: Adolescent; Adolescent behavior; Health; Nursing.

\title{
Resumen
}

Objetivo: Identificar cómo los adolescentes interpretan y reaccionan a sus relaciones amistosas y cuáles son sus repercusiones en el bienestar y la salud en esta etapa de la vida. Método: Este es un estudio transversal con un enfoque cualitativo, exploratorio y descriptivo, 
efectuado entre junio y julio de 2019. El escenario de investigación fue una escuela ubicada en un ayuntamiento en el extremo sur del estado de Rio Grande do Sul, que se dedica a la oferta de cursos preprofesionales a estudiantes adolescentes en situaciones de vulnerabilidad social, económica y ambiental. Resultados: El estudio tuvo la participación de 30 adolescentes. Después de analizar los temas originados en las entrevistas, surgieron las siguientes categorías: Amistades saludables y promotoras de afecto y empatía; Confianza: la necesidad de una base segura en las interacciones y Amistades disfuncionales: un riesgo para la salud de los adolescentes. Consideraciones Finales: Las relaciones interpersonales durante la adolescencia están impregnadas de singularidades y sutilezas, donde los individuos son más sensibles a las críticas y la desaprobación de sus pares. Las actitudes negativas de rechazo y negligencia tienen el carácter de una mayor exposición a comportamientos que comprometen en gran medida su desarrollo saludable.

Palabras clave: Adolescent; Conducta del adolescent; Salud; Enfermeria.

\section{Introdução}

O comportamento dos adolescentes diante dos contextos de vida é produzido consciente ou inconscientemente pelas interações estabelecidas com o meio, de acordo com seu tempo, cultura e ambiente social. As pessoas que vivem e convivem ao seu redor, seja no ambiente familiar, os amigos ou colegas constituem seu universo de troca e aprendizado. Neles, as experiências compartilhadas, positivas ou negativas, terão repercussões sobre suas escolhas e inevitavelmente sobre sua saúde.

O suporte e a qualidade das amizades são indicativos para o funcionamento psicológico dos adolescentes. As amizades evoluem para relacionamentos mais íntimos, de apoio e lealdade. Ter amigos e ser apreciado por eles reforça a satisfação pessoal, fornece confiança no futuro e melhora a capacidade de responder às dificuldades cotidianas. Uma boa relação com os amigos contextualiza um ambiente apropriado para o desenvolvimento de estratégias eficazes, resolução de problemas e transformação de ideias em projetos (Fadda, Scalas \& Meleddu, 2015).

No momento em que as crianças se tornam adolescentes, os colegas tornam-se mais importantes no desenvolvimento cognitivo, social e emocional dos jovens e constituem fonte de influência social no ajustamento dos adolescentes. Os pares são indivíduos que compartilham alguma equidade relativa em relação à idade, status dentro de contextos sociais que ocupam como por exemplo, escolas, vizinhanças e redes sociais on-line. As amizades são 
interações voluntárias e recíprocas e seu propósito é satisfazer necessidades socioafetivas de amor, apego, afeto, intimidade, lealdade, apoio e segurança. (Véronneau, Trempe \& Paiva, 2014).

Problemas como a solidão, ansiedade, depressão e baixa autoestima podem ser observados entre adolescentes que sofrem de vitimização por seus pares, como por exemplo, boatos espalhados e ser excluído de um grupo. Este tipo de interação social disfuncional entre pares dificulta a saúde e o desenvolvimento psicossocial, aumentando os problemas de internalização em adolescentes, sendo considerada um importante risco à saúde e não um aspecto tolerável da vida do adolescente. Atualmente a arena social dos adolescentes é muito mais complexa e inclui tanto interações reais quanto online, ambas têm um significado diferenciado e impacto na saúde e no bem-estar dos adolescentes (Eijnden et al., 2014).

A influência que os pares podem exercer no comportamento do adolescente é uma questão complexa, devido à variedade de contextos que envolve sua a rede de amizades e sua capacidade de interagir com o mundo ao seu redor. Perante ao exposto, o objetivo do estudo foi identificar como os adolescentes interpretam e reagem diante de suas relações de amizade e quais suas repercussões no bem-estar e saúde nesta fase da vida.

\section{Metodologia}

Trata-se um estudo transversal de abordagem qualitativa, exploratório e descritivo realizado no período de junho a julho de 2019. O cenário da pesquisa foi uma escola localizada em município do extremo sul do estado do Rio Grande do Sul, que se dedica a oferta de cursos pré-profissionalizantes a estudantes adolescentes em situação de vulnerabilidade social, econômica e ambiental.

Os estudantes foram apresentados a pesquisa e convidados a participar. No entanto foram incluídos apenas aqueles que assinaram os termos de assentimento e receberam o consentimento dos pais ou responsáveis autorizando sua participação. Os adolescentes em que houve discordância entre pais e filhos na apresentação dos termos foram excluídos, assim como ausência em sala de aula no dia da entrevista. Os estudantes são distribuídos em 15 turmas e a seleção da amostra deu-se por sorteio entre os interessados, desta forma dois de cada turma totalizando 30 participantes.

O número de participantes estabelecido, corresponde ao quantitativo máximo recomendado para uma amostra qualitativa, refletindo em quantidade e intensidade, as múltiplas dimensões do fenômeno estudado, buscando a qualidade das interações em todo o 
decorrer do processo da investigação qualitativa (Minayo, 2017).

A coleta dos dados foi realizada por meio de entrevista semiestruturada composta por questões norteadoras, com duração média de 30 minutos. Aconteceram no período das aulas, em ambiente reservado, garantindo a privacidade dos adolescentes e respeitando a possibilidade de recusa em permanecer no estudo se assim fosse desejado por eles. No entanto, não obtivemos nenhum declínio quanto ao envolvimento no estudo. Para assegurar o sigilo e anonimato, os adolescentes foram identificados com a letra A seguida do número da entrevista concedida pela ordem em que ocorreram, ou seja, de 1 a 30 .

O número de participantes foi estabelecido previamente, atingindo o quantitativo máximo de 30 entrevistas recomendado para uma amostra qualitativa, refletindo em quantidade e intensidade, as múltiplas dimensões do fenômeno estudado, buscando a qualidade das interações em todo o decorrer do processo da investigação qualitativa (Minayo, 2017).

Os adolescentes foram questionados sobre sua percepção sobre seu relacionamento com amigos, e as entrevistas foram analisadas pela técnica de análise temática, operacionalizada a partir de uma pré-análise dos dados, na qual foi realizado o agrupamento das falas e elaboração das unidades de registro; exploração do material, na qual os dados foram codificados, agrupados por semelhanças, diferenças, organizados em categorias e interpretação dos resultados obtidos. Foram selecionadas as falas mais significativas para ilustrar a análise e realizar a busca de autores para dar suporte à análise (Minayo, 2012).

O presente estudo recebeu aprovação do Comité de Ética e Pesquisa na Área da Saúde da Universidade Federal do Rio Grande - FURG, parecer $n^{\circ}$ 130/2019, CAAE 96938118.0.0000.5324, atendendo desta forma, as exigências da Resolução nº 466/2012, do Conselho Nacional de Saúde.

\section{Resultados e Discussão}

Os participantes tinham entre 14 e 17 anos, sendo a média de idade 15,5 anos; a maioria composta por adolescentes do sexo feminino $63 \%$ e cursando o ensino fundamental cerca de $76 \%$. Após análise dos temas originados nas entrevistas emergiram as seguintes categorias: Relações de amizade saudáveis e promotoras de afeto, empatia e confiança; Confiança: a necessidade de uma base segura nas interações e Amizades disfuncionais: um risco para a saúde do adolescente. 


\subsection{Relações de amizade saudáveis e promotoras de afeto e empatia}

Alguns adolescentes vivenciam relações saudáveis de amizade. Colocam-se como bons amigos de seus amigos buscando transparecer a sensibilidade em oferecer e receber carinho, afeto, estando disponíveis para compartilhar tanto os momentos de diversão quanto os de dificuldade. Percebemos o valor das amizades na vida destes adolescentes e a maneira positiva e otimista de perceber as relações. Demonstrações de empatia e compreensão pela condição do outro são identificadas nas interações com os amigos.

Meus amigos, eu trato eles com amor com carinho e trato eles do jeito que eu gosto de ser tratado tipo eu abraço, dou beijo na bochecha, essas coisas, sabe. (A3)

Me sinto feliz com minhas amizades, me dou bem com todo mundo. Eu sou muito comunicativa e muito carinhosa. Faço bastante amizade em todos lugares que frequento, em cursos, no colégio. (A5)

Eu sempre me dou bem com todo mundo. Nunca briguei com ninguém porque eu entendo as pessoas, sei lá. Eu já passei por tanta coisa que eu não consigo passar negatividade para as pessoas. Procuro entender elas, sentar para conversar, saber o que está se passando e o que me contam eu não conto nada para ninguém. Eu acho que isso não é ser ético né. Eu tento ajudar da maneira que eu posso sempre que elas precisam de mim. (A21)

\subsection{Confiança: a necessidade de uma base segura nas interações}

A confiança foi considerada pelos adolescentes como uma característica essencial em uma relação de amizade e assumem que sua ausência interfere na aproximação e manutenção dos amigos. A relação com a mãe, foi considerada a de maior confiança pelos adolescentes.

Eu sou uma pessoa que tem um problema, eu preciso de atenção e confiança. Se minhas amigas não me passam essa firmeza, eu fico mal, não é suficiente para mim. Eu chego em casa e choro. Então, é uma coisa do tipo: fala comigo, senão não dá!! Não consigo ver as pessoas que eu gosto sem estarem fechadas comigo, sabe. (A6) 
Research, Society and Development, v. 9, n. 8, e105985363, 2020

(CC BY 4.0) | ISSN 2525-3409 | DOI: http://dx.doi.org/10.33448/rsd-v9i8.5363

É digamos, eu não tenho amigos. Só sou eu, minha mãe e meu namorado, bem dizer, o meu particular eu falo para eles. Não conto as minhas histórias, a minha vida para amigos, entendeu? Porque a gente nunca sabe quem é realmente amigo, quem não é. (A8)

Estou sempre brincando com elas só que tem umas gurias que são meio bipolar, sabe, e eu não dou muito papo. Ficam de cara amarrada sem me dizer o motivo daí eu fico na minha e a amizade enfrequece. Amiga não vou dizer que tenho, tenho colegas, amiga é minha mãe. (A17)

Não sei se tenho amigos de verdade. Eu até consigo fazer amizade fácil, só que eu não consigo confiar nas pessoas. Então, eu tenho colegas apenas. (A18)

Tenho poucos amigos. Sabe porquê? Para ver se é amigo mesmo tem que ter uma situação crítica e ver o que ela vai fazer, porque só uma situação da corda bamba é que a gente vê quem são os amigos de verdade. Eu acho que pessoas que eu poderia contar mesmo são duas. Elas estão sempre comigo, querendo saber se eu estou bem. (A23)

\subsection{Amizades disfuncionais: um risco para a saúde do adolescente}

Quando os conflitos e a falta de apoio nas relações de amizade se fazem presentes, os adolescentes são tomados por um abalo emocional. Afetando suas formas de encarar a vida e as relações. Sentimentos de solidão e falta de empatia levaram a sintomas depressivos como consequência de situações adversas enfrentadas no contexto das amizades.

Eu sentia meio que um vazio, não tem como explicar direito. Foi meio que do nada eu comecei a olhar a minha volta e perceber o que estava acontecendo. Eu estava sozinho, percebi que eu não tinha tantos amigos e que eles não estavam ligando muito para mim, percebi que não eram verdadeiras as amizades. Me isolaram, me senti abandonado sem ninguém do lado para desabafar. (A30)

Quando meus amigos falam ou me dão características que eu não sou fico magoada profundamente; e teve um tempo que eu fiquei muito abalada. Em vez de virem falar comigo e dizer que eu tinha que mudar eles ficavam falando muita coisa para outras 
Research, Society and Development, v. 9, n. 8, e105985363, 2020

(CC BY 4.0) | ISSN 2525-3409 | DOI: http://dx.doi.org/10.33448/rsd-v9i8.5363

pessoas, acho isso injusto. Perdi muitas amizades por isso, as pessoas se afastaram de mim, me sentia sozinha e triste... vivia chorando sem vontade de fazer nada. (A7)

Eu tinha uma amizade, e por muitas mentiras que uma guria inventou a gente parou de se falar. Nesse tempo eu emagreci dez quilos num mês, eu tive tipo uma depressão sabe. (A16)

Nem todas as amizades se constroem da mesma maneira. Existem aquelas de qualidade superior marcadas pela lealdade, confiança, reciprocidade, companheirismo, confiança e suporte emocional. Por outro lado, encontram-se amizades de qualidade inferior onde a rivalidade, o conflito, a dominância e a crítica estão presentes (Freitas et al., 2015). A capacidade de construção e manutenção de relações de amizade satisfatórias constitui-se como um indicador fidedigno da saúde física e mental do adolescente, além de ser um fator psicossocial protetor, estando associado a níveis superiores de autoestima e bem-estar. Esses efeitos também se expressam na vida adulta em diferentes ambientes, ou seja, influenciam nos contextos sociais, acadêmicos e de trabalho.

Existe uma relação de interdependência entre a aceitação dos amigos e o autoconceito social positivo (Carvalho et al., 2017). Nesta fase da vida, o critério qualidade das amizades permite ao adolescente identificar as particularidades dessa relação e os respectivos recursos existentes ou ausentes na mesma. Por isto a confiança nos amigos é citada como uma característica importante.

Adolescentes com níveis elevados de satisfação e gratidão pela interação social otimista com seus pares, desfrutam dos benefícios contribuídos por eles para as suas experiências pessoais. As emoções positivas têm um impacto na felicidade do adolescente, pois envolve reflexão, comportamentos sociais adaptativos e relacionamentos que facilitam o bem-estar o que por sua vez diminuem as experiências de raiva, sentimentos de solidão e sintomas depressivos (Reckart et al., 2017 ; Rey et al., 2019).

A prevalência de solidão na adolescência é alta, variando entre $21 \%$ a $70 \%$ de adolescentes sentindo-se solitários pelo menos algumas vezes e entre 3 e $22 \%$ de adolescentes experimentando cronicamente a solidão. Ela pode ser definida como uma experiência subjetiva de falta de conexão, em termos de quantidade ou qualidade das relações sociais. Tem sempre consequências negativas para a saúde mental e física, incluindo depressão, ideação suicida, agressão, obesidade e doenças cardiovasculares aumentando o risco de mortalidade precoce (Lodder et al., 2016). 
A solidão afeta as habilidades sociais dos adolescentes, dificultando a capacidade de interagir com outras pessoas, limitando sua oportunidade de formar e manter amizades satisfatórias além de demonstrar dificuldade em lidar adequadamente com eventos estressantes ao envolver sua rede social, levando ao aumento de sentimentos negativos como a tristeza e falta de esperança nas relações futuras.

As amizades reservam um importante lugar nas interações sociais dos adolescentes. Quando associadas a sentimentos frustrantes e conflitantes podem levar a sintomas depressivos pelo medo da avaliação negativa por seus pares. (Kornienko \& Santos, 2014). Na adolescência, a pessoa com depressão é comumente representada como aquela que vivencia carência afetiva, a exemplo de não possuir amigos, sentimento de rejeição, solidão, baixa autoestima, falta de amor, desvalorização e isolamento social. (Coutinho et al., 2016).

Considerada um fenômeno complexo e multidimensional, a depressão afeta diretamente a saúde mental, a qualidade de vida e a vida social do indivíduo como um todo. Trata-se de um dos problemas de saúde mais prevalentes em todo o mundo, acarretando isolamento social, constituindo fator de risco para suicídios. Segundo a OMS, a depressão está entre as doenças mais comuns e pode acometer pessoas em qualquer fase da vida, no entanto, há indicativos de aumento significativos desse transtorno durante a adolescência e no início da vida adulta, sendo mais comum no sexo feminino (Gavin et al, 2015).

No entanto, independentemente do sexo ou estágio da adolescência, os adultos podem assumir o papel, em diferentes contextos sociais, de incentivadores de atividades humanitárias, trabalhos sociais e voluntariado como estratégias para promover um clima que facilite o desenvolvimento de virtudes e comportamentos pró-sociais nos adolescentes, evitando que eles destaquem as fraquezas dos colegas ou amigos (García-Vázquez et al., 2020).

\section{Considerações Finais}

A partir do exposto, pode-se identificar que no período da adolescência, abrem-se novos espaços de experimentação e de interação no contexto das amizades, os quais contribuem para que cada sujeito possa se conhecer na sua singularidade. Os amigos têm papel fundamental na construção da socialização. As relações estabelecidas na adolescência influenciam as percepções do adolescente sobre si mesmos nesta e em outras fases da vida.

Podemos identificar que a forma de se comunicar do adolescente com o mundo ao seu redor desencadeia manifestações nas dimensões internas e externas. Internamente observa-se 
que as experiências influenciam as necessidades emocionais. Eles precisam de atenção, confiança, reconhecimento, pertencimento e aceitação. Externamente, identificou-se que o comportamento é influenciado por estas emoções. Observou-se que a rejeição, os conflitos, as agressões verbais, a intimidação, a tristeza e a desilusão foram geradas a partir de necessidades suprimidas ou negligenciadas por seus pares.

O presente estudo demonstrou que as relações interpessoais são permeadas de singularidades e sutilezas. Durante a adolescência os indivíduos estão mais sensíveis a críticas e desaprovação por seus pares. Atitudes negativas de rejeição e negligência tem caráter de maior exposição a comportamentos de isolamento social, traduzidos pelos adolescentes pela acentuada tristeza e sintomas depressivos, o que compromete sobremaneira seu desenvolvimento saudável.

\section{Referências}

Brasil. (2012) Conselho Nacional de Saúde. Resolução nº 466, de 12 de dezembro de 2012. Brasília - DF 2012. Disponível em: http://www.conselho.saude.gov.br/web_comissoes/conep/ index.html.

Carvalho, R. G., et al. (2017). Relations of friendship and self-concept in adolescence: An exploratory study in the school contexto. Estudos de Psicologia. 34(3) I 379-388. doi: http://dx.doi.org/10.1590/1982-02752017000300006.

Coutinho, M. P. L., et al. (2016). Relação entre depressão e qualidade de vida de adolescentes no contexto escolar. Psic., Saúde \& doenças. (17):3. Lisboa. doi: http://dx.doi.org/10.15309/ 16 psd1 70303

Eijnden, V. D. R., et al. (2014). The Bidirectional Relationships Between Online Victimization and Psychosocial Problems in Adolescents: A Comparison with Real-Life Victimization. J Youth Adolescence 43,790-802. doi: https://doi.org/10.1007/s10964-0130003-9

Fadda, D., Scalas, L. F., \& Meleddu, M. (2015). Contribution of personal and environmental factors on positive psychological functioning in adolescents. Journal of Adolescence. (43), p. 119-131. doi: https://doi.org/10.1016/j.adolescence.2015.05.019. 
Freitas, M, et al. (2015). Análise factorial confirmatória do modelo do Questionário da Qualidade da Amizade numa amostra de jovens adolescentes Portuguesa. Laboratório de Psicologia, 11(2), 163-175. doi: https://doi. org/10.14417/lp655

García-Vázquez, F. I., et al. (2020). Forgiveness, Gratitude, Happiness, and Prosocial Bystander Behavior in Bullying. Frontiers in psychology, 10, 2827. doi: https://doi.org/10.3389/fpsyg.2019.02827

Gavin, R., et al. (2015). Associação entre depressão, estresse, ansiedade e uso de álcool entre servidores públicos. SMAD. Revista Eletrônica Saúde Mental Álcool e Drogas. 11(1), 2-9. doi: http://dx.doi.org/10.11606/issn.1806-6976.v11i1p2-9.

Kornienko, O., \& Santos, C. E. (2014). The effects of friendship network popularity on depressive symptoms during early adolescence: Moderation by fear of negative evaluation and gender. Journal of Youth and Adolescence. (43), p. 541- 553. doi: http://dx.doi.org/10.1007/s10964-013-9979-4.

Lodder, G. M. A, et al. (2016). Adolescent loneliness and social skills: Agreement and discrepancies between self-, meta-, and peer-evaluations. Journal of Youth and Adolescence . (45), 2406-2416. doi: https://doi.org/10.1007/s10964-016-0461-y.

Minayo, M. C. S. (2012) Análise qualitativa: teoria, passos e fidedignidade. Ciênc. saúde coletiva, 17(3),.621-626. doi: http://www.scielo.br/scielo.php?pid=S1413$81232012000300007 \&$ script=sci_abstract\&tlng=pt.

Minayo, M. C. S. (2017). Amostragem e saturação em pesquisa qualitativa: consensos e controvérsias. Revista Pesquisa Qualitativa. São Paulo, 5(7) p. 01-12.

Reckart, H., et al. (2017). A preliminary study of the origins of early adolescent gratitude differences. Pers. Indiv. Differ. doi:116 44-50. 10.1016/j.paid.2017.04.020.

Rey, L., et al. (2019). Being bullied at school: gratitude as potential protective factor for suicide risk in adolescents. Front. Psychol. doi: 10:662. 10.3389/fpsyg.2019.00662. 
Veronneau, M. H., Trempe, S. C., \& Paiva, A. O. (2014). Risk and protection factors in the peer context: how do other children contribute to the psychosocial adjustment of the adolescent? Ciênc. saúde coletiva. 19(3), 695-705. doi: http://dx.doi.org/10.1590/141381232014193.17972013.

\section{Porcentagem de contribuição de cada autor no manuscrito}

Eloisa da Fonseca Rodrigues - 20\%

Giovana Calcagno Gomes - 20\%

Luciano Garcia Lourenção - $15 \%$

Simone Quadros Alvarez - 15\%

Aline Campelo Pintanel - 15\%

Juliane Portella Ribeiro - 15\% 